\title{
Heart failure and clinical practice
}

\author{
Giorgio Costantino $\cdot$ Anna Maria Rusconi • \\ Stefano Guzzetti $\cdot$ Nicola Montano
}

Received: 12 August 2009/Accepted: 21 August 2009/Published online: 12 September 2009

(C) SIMI 2009

The interesting letter by Bruno Trimarco and Alessandro Bellis regarding our study [1] opens two complex questions. First, the capacity of a randomized clinical trial (RCT) to represent the real world, and, when it does, what is the possibility of obtaining reliable results? This produces a need for a delicate balance. If the selection of patients in a RCT study is tight, less "confounders" can influence the results, but the results can be applicable to only a limited number of patients. A theoretical solution for this first point is, in our opinion, impossible. In our study [1] we tried only to highlight that in most of the major heart failure RCT studies, published in prior years, a tight selection of a particular type of patients (relatively young, male, without comorbidities, etc.) has been often the chosen study group. This selection reduces the capacity of these studies to represent our general population of patients with heart failure. We completely agree with Trimarco and Bellis that: "the guidelines for management of heart failure (resulting from RCT results) should be interpreted rather than strictly followed". Nevertheless, if the results were obtained from a selected population of patients with heart failure, we do not have reliable indications for many other patients (old, female; with comorbidities, etc.), in particular for treatment choices. Treating patients on the basis of "pathophysiological observations", without consistent results from RCT studies, is considered a "second-choice" practice by many clinicians.

The other point raised by Trimarco and Bellis [2] concerns the observation that mortality in heart failure trials is reduced only for a brief follow-up period (no more than 5 years), whereas when longer observational periods are considered, treatment efficacy is progressively reduced.

In this regard we would like to emphasize that heart failure prognosis is ominous. The 1-year mortality is more than $20 \%$ [3], while the 5-year mortality more than $70 \%$ [3]. Stewart and colleagues [3] compare heart failure prognosis with breast, bowel, lung and ovarian cancers and they observe that only lung cancer prognosis is worse than that of heart failure. We think that clinical trials with a less selected population, and with a 2-3 years period of followup could be more similar to everyday clinical practice, and could offer an answer to the real efficacy of heart failure therapy. Because of the ominous prognosis connected to heart failure, a longer follow-up could mask treatments effects without giving more informative results.

Conflict of interest statement The authors declare that they have no conflict of interest related to the publication of this manuscript.

\section{References}

1. Costantino G, Rusconi AM, Duca PG et al (2009) Eligibility criteria in heart failure randomized controlled trials: a gap between evidence and clinical practice. Intern Emerg Med 4:117-122

2. Trimarco B, Bellis A (2009) Clinical trials in heart failure: may we trust? Intern Emerg Med. doi:10.1007/s11739-009-0275-y

3. Stewart S, MacIntyre K, Hole DJ et al (2001) More "malignant" than cancer? Five-year survival following a first admission for heart failure. Eur J Heart Fail 3:315-322 